\title{
On $p$-nilpotency of finite group with normally embedded maximal subgroups of some Sylow subgroups
}

\section{A. Trofimuk}

\author{
Communicated by A. Yu. Olshanskii
}

\begin{abstract}
A Bstract. Let $G$ be a finite group and $P$ be a $p$-subgroup of $G$. If $P$ is a Sylow subgroup of some normal subgroup of $G$, then we say that $P$ is normally embedded in $G$. Groups with normally embedded maximal subgroups of Sylow $p$-subgroup, where $(|G|, p-1)=1$, are studied. In particular, the $p$-nilpotency of such groups is proved.
\end{abstract}

\section{Introduction}

All groups considered in this paper will be finite. Our notation is standard and taken mainly from [1], [2].

Let $\mathcal{M}(G)$ be the set of all maximal subgroups of Sylow subgroups of a group $G$. One of the first results related to the study of the structure of a group with given restrictions on $\mathcal{M}(G)$ belongs to Srinivasan, see [3]. In particular, in [3] it is proved that a group $G$ is supersolvable, if every subgroup of $\mathcal{M}(G)$ is normal in $G$. Subsequently, groups with restrictions on subgroups of $\mathcal{M}(G)$ have been studied in the works of many authors, see the literature in [4].

A subgroup $H$ of $G$ is said to be $S$-embedded in $G$, see [5], if $G$ has a normal subgroup $N$ such that $H N$ is $S$-permutable in $G$ and

2010 MSC: 20D10.

Key words and phrases: $p$-supersolvable group, normally embedded subgroup, maximal subgroup, Sylow subgroup. 
$H \cap N \leqslant H_{s G}$, where $H_{s G}$ is the largest $S$-permutable subgroup of $G$ contained in $H$. In the paper [5] the structure of the groups depending on $S$-embedded subgroups is studied. In particular, the $p$-nilpotency of a group $G$ for which every subgroup of $\mathcal{M}(P)$ is $S$-embedded in $G$, where $P$ is a Sylow $p$-subgroup of $G$ and $p \in \pi(G)$ such that $(|G|, p-1)=1$ follows from [5, Theorem 2.3].

In the present paper, we study another generalization of normality.

Definition. A subgroup $H$ of a group $G$ is said normally embedded in $G$, if for every Sylow subgroup $P$ of $H$, there is a normal subgroup $K$ of $G$ such that $P$ is Sylow subgroup of $K$, see [6, I.7.1].

A series of results related to the structure of a group with normally embedded subgroups is presented in [6].

The following examples show that $S$-embedded and normally embedded are different concepts.

In the symmetric group $S_{5}$ of degree 5 some maximal subgroup $H$ of a Sylow 2-subgroup is a Sylow 2-subgroup in the normal alternating subgroup $A_{5}$ of degree 5, i.e. $H$ is normally embedded in $S_{5}$. But, $H$ is not $S$-embedded. In the alternating group $A_{4}$ of degree 4 some maximal subgroup $M$ of a Sylow 2-subgroup is not normally embedded in $A_{4}$. But, $M$ is $S$-embedded.

In this paper, the structure of a group $G$ under the condition that every subgroup of $\mathcal{M}(P)$ is normally embedded in $G$ is studied, where $P$ is a Sylow $p$-subgroup of $G$ and $p \in \pi(G)$ such that $(|G|, p-1)=1$.

The following theorem is proved.

Theorem. Let $G$ be a group, $H$ be a normal subgroup of $G$ such that $G / H$ is p-nilpotent and $P$ be a Sylow p-subgroup of $H$, where $p \in \pi(G)$ with $(|G|, p-1)=1$. If every subgroup of $\mathcal{M}(P)$ is normally embedded in $G$, then $G$ is p-nilpotent.

\section{Preliminaries}

In this section we collect lemmas used in the proof of the main theorem presented in Section 2.

The Fitting subgroup and the Frattini subgroup of $G$ are denoted by $F(G)$ and $\Phi(G)$, respectively; we write $Z_{m}$ for a cyclic group of orders $m$; $\mathrm{O}_{p}(G)$ and $\mathrm{O}_{p^{\prime}}(G)$ denote the greatest normal $p$-subgroup of $G$ and the greatest normal $p^{\prime}$-subgroup of $G$, respectively. By $\pi(G)$ denote the set 
of all prime divisors of the order of $G$; by $H^{G}$ denote the normal closure of a subgroup $H$ in a group $G$, i.e. the smallest normal subgroup of $G$ containing $H$. We write $H$ ne $G$ for normally embedded subgroup $H$ of $G$ and $G=[A] B$ for the semidirect product of some subgroups $A$ and $B$ with the normal subgroup $A$.

If the orders of chief factors of $G$ are either equal to $p$ or not divisible on $p$ then $G$ is called $p$-supersolvable. We denote by $p \mathfrak{U}$ the class of all $p$-supersolvable groups. A group that has a normal Sylow $p$-subgroup is called $p$-closed and a group that has a normal $p^{\prime}$-Hall subgroup is called $p$-nilpotent.

Let $G$ be a group of order $p_{1}^{a_{1}} p_{2}^{a_{2}} \ldots p_{k}^{a_{k}}$, where $p_{1}>p_{2}>\ldots>p_{k}$. We say that $G$ has an ordered Sylow tower of supersolvable type if there exists a series

$$
1=G_{0}<G_{1}<G_{2}<\ldots<G_{k-1}<G_{k}=G
$$

of normal subgroups of $G$ such that $G_{i} / G_{i-1}$ is isomorphic to a Sylow $p_{i}$-subgroup of $G$ for each $i=1,2, \ldots, k$.

Lemma 1 ([6, I.7.3]). Let $U$ be a normally embedded p-subgroup of a group $G, K$ a normal subgroup of $G$. Then:

(1) if $U \leqslant H \leqslant G$, then $U$ ne $H$;

(2) $U K / K$ ne $G / K$;

(3) $U \cap K$ ne $G$;

(4) if $K$ is a p-group, then $U K$ ne $G$ and $U \cap K$ is normal in $G$;

(5) $U^{g}$ ne $H$ for all $g \in G$.

Lemma 2. Let $H$ be a normal subgroup of $G$ and every maximal subgroup of Sylow p-subgroup of $H$ is normally embedded in $G$. If $N$ is normal in $G$, then every maximal subgroup of every Sylow p-subgroup of $H N / N$ is normally embedded in $G / N$. In particular, if $N$ is normal in $G$ and every maximal subgroup of Sylow p-subgroup of $G$ is normally embedded in $G$, then every maximal subgroup of every Sylow p-subgroup of $G / N$ is normally embedded in $G / N$.

Proof. By Lemma 1(5), it follows that $X_{1}$ is normally embedded in $G$ for any Sylow $p$-subgroup $X$ of $H$ and any maximal subgroup $X_{1}$ of $X$. Let $\overline{P_{1}}=X / N$ is a maximal subgroup of Sylow $p$-subgroup $\bar{P}$ of $H N / N$. Then $N \leqslant X \leqslant H N$ and there exists a Sylow $p$-subgroup $P$ in $H N$ such that $\bar{P}=P N / N$. By [1, VI.4.6], there exist the Sylow $p$-subgroups $H_{p}$ in $H$ and $N_{p}$ in $N$ such that $P=H_{p} N_{p}$, hence $\bar{P}=H_{p} N / N$. Further, 
$N \leqslant X<P N \leqslant H_{p} N$ and $X=\left(X \cap H_{p}\right) N$ by Dedekind's identity. Since $H_{p} \cap N=X \cap H_{p} \cap N$, we have

$$
\begin{aligned}
p & =\left|\bar{P}: \overline{P_{1}}\right|=\left|H_{p} N / N: X / N\right|=\left|H_{p} N: X\right| \\
& =\left|H_{p} N:\left(X \cap H_{p}\right) N\right|=\frac{\left|H_{p}\right||N|\left|X \cap H_{p} \cap N\right|}{\left|H_{p} \cap N\right|\left|X \cap H_{p}\right||N|}=\left|H_{p}: X \cap H_{p}\right| .
\end{aligned}
$$

So, $X \cap H_{p}$ is a maximal subgroup in $H_{p}$. By hypothesis, $X \cap H_{p}$ is normally embedded in $G$. By Lemma $1(2),\left(X \cap H_{p}\right) N / N=X / N$ is normally embedded in $G / N$.

For $H=G$ we obtain the second part of the lemma.

Lemma 3 ([7, Lemma 5]). Let $G$ be a p-solvable group. Assume that $G$ does not belong to $p \mathfrak{U}$, but $G / K \in p \mathfrak{U}$ for all non-trivial normal subgroups $K$ of $G$. Then:

(1) $Z(G)=\mathrm{O}_{p^{\prime}}(G)=\Phi(G)=1$;

(2) $G$ contains a unique minimal normal subgroup $N, N=F(G)=$ $\mathrm{O}_{p}(G)=C_{G}(N)$

(3) $G$ is primitive; $G=[N] M$, where $M$ is maximal in $G$ with trivial core;

(4) $N$ is an elementary Abelian subgroup of order $p^{n}, n>1$;

(5) if $M$ is Abelian, then $M$ is cyclic of order dividing $p^{n}-1$, and $n$ is the smallest natural number such that $p^{n} \equiv 1(\bmod |M|)$.

A non-nilpotent group whose proper subgroups are all nilpotent is called a Schmidt group.

Lemma 4 ([8]). Let $S$ be a Schmidt group. Then:

(1) $S=[P] Q$, where $P$ is a normal Sylow p-subgroup, $Q$ is a non-normal Sylow $q$-subgroup, $p$ and $q$ are distinct primes;

(2) $Q=\langle y\rangle$ is cyclic and $y^{q} \in Z(S)$;

(3) $\left|P / P^{\prime}\right|=p^{m}$, where $m$ is the order of $p$ modulo $q$;

(4) the chief series of $S$ has the following system of indexes: $p, p, \ldots, p$, $p^{m}, q, \ldots, q$; number of indexes equal to $p$ coincides with $n$, where $p^{n}=\left|P^{\prime}\right|$; number of indexes equal to $q$ coincides with $b$, where $q^{b}=|Q|$.

Lemma 5. Let $p \in \pi(G)$ and $(|G|, p-1)=1$. Then $G$ is $p$-supersolvable if and only if $G$ is p-nilpotent. In particular, if a Sylow p-subgroup is cyclic, then $G$ is p-nilpotent. 
Proof. It is clear that every $p$-nilpotent group is $p$-supersolvable. Conversely. Let $G$ be a group of the smallest order such that $G$ is $p$-supersolvable, but is not $p$-nilpotent. Let $H$ be an arbitrary proper subgroup of $G$. Then $H$ is $p$-supersolvable and $(|H|, p-1)=1$. Therefore in view of the choice $G$, the subgroup $H$ is $p$-nilpotent and $G$ is a minimal non$p$-nilpotent group. By [9, Theorem 10.3.3], $G$ is a Schmidt group. By Lemma $4(1), G=[P] Q$, where $P$ is a Sylow $p$-subgroup and $Q$ is a cyclic Sylow $q$-subgroup. Since $G$ is $p$-supersolvable, then by Lemma 4 (4), the order of $p$ modulo $q$ is equal 1 , i.e. $m=1$. Hence $q$ divides $p-1$. This is a contradiction.

In particular, if a Sylow $p$-subgroup is cyclic, then $G$ is $p$-supersolvable. Then $G$ is $p$-nilpotent by what has been proved above. The lemma is proved.

Corollary 1. Let $p$ be the smallest prime of $\pi(G)$. Then $G$ is p-supersolvable if and only if $G$ is p-nilpotent.

Example 1. The symmetric group $G=S_{3}$ of degree 3 is 3 -supersolvable, but is not 3-nilpotent. Hence, the condition $(|G|, p-1)=1$ in Lemma 5 can not be removed.

Example 2. A group $G=Z_{5} \times\left(\left[Z_{7}\right] Z_{3}\right)$ is 5-supersolvable and is 5nilpotent. In addition, $(|G|, 5-1)=1$, and the prime divisor 5 of $|G|$ is not the smallest.

Evidently, if a $p$-subgroup $P$ of $G$ is normally embedded in $G$, then $P$ is a Sylow subgroup of $P^{G}$.

Lemma 6. Let $G$ be a group, $\Phi(G)=1, P$ be a Sylow subgroup of $G$ with unprimary order and $N$ be a unique minimal normal subgroup of $G$. If every subgroup of $\mathcal{M}(P)$ is normally embedded in $G$ and $N$ is Abelian, then $N$ is not contained in $P$.

Proof. Suppose that $N \leqslant P$. If $N=P$, then by hypothesis, every maximal subgroup $S$ of $P$ is normally embedded in $G$. Then by Lemma 1(4), $S$ is normal in $G$. Since the order of $P$ is not equal to a prime, we have a contradiction with the fact that $N$ is a minimal normal subgroup in $G$.

In the following we assume that $N<P$. Since $\Phi(G)=1$, it follows that there exists a maximal subgroup $M$ of $G$ such that $N$ is not contained in $M$. Hence $G=N M$. By [2, Lemma 2.36], $N \cap M=1$ and $G=[N] M$. Then by Dedekind's identity, $P=P \cap[N] M=[N](P \cap M)$, where 
$P \cap M \neq 1$. Let $T$ be a maximal subgroup of $P$ such that $P \cap M \leqslant T$. Since $N$ is a unique minimal normal subgroup of $G$, it follows that $N \leqslant T^{G}$. Now, $P=N T \leqslant T^{G}$, but by hypothesis, $T$ is a Sylow subgroup of $T^{G}$, a contradiction.

Lemma 7. Let $P$ be a Sylow p-subgroup of $G$. If every subgroup of $\mathcal{M}(P)$ is normally embedded in $G$ and $(|G|, p-1)=1$, then $G$ is p-nilpotent.

Proof. We use induction on the order of $G$. Since $(|G / N|, p-1)=1$ and by Lemma 2, every maximal subgroup of every Sylow $p$-subgroup of $G / N$ is normally embedded in $G / N$ for any normal subgroup $N \neq 1$ of $G$, then all quotients of $G$ satisfy the hypotheses of the lemma.

By the inductive hypothesis, $O_{p^{\prime}}(G)=1$. Since the class of all $p$ nilpotent groups is a saturated formation, then $\Phi(G)=1$ and $N=$ $F(G)=O_{p}(G)$ is a unique minimal normal subgroup $G$. Hence there is a Sylow $p$-subgroup $R$ of $G$ such that $N \subseteq R$. Since $R$ and $P$ are conjugate in $G$, then by Lemma 1(5), it follows that every maximal subgroup of $R$ is normally embedded in $G$. If $|R|=p$, then $G$ is $p$-nilpotent by Lemma 5 . Therefore, we further assume that $|R|>p$. By Lemma $6, N$ is not contained in $R$. This is a contradiction. The lemma is proved.

\section{Proof of the theorem}

In view of Lemma 5 , we prove that $G$ is $p$-supersolvable.

By Lemma 1(1), every maximal subgroup of Sylow $p$-subgroup $P$ of $H$ is normally embedded in $H$ and $(|H|, p-1)=1$. By Lemma $7, H$ is $p$-nilpotent. Since by hypothesis, $G / H$ is $p$-nilpotent, then $G$ is $p$-solvable.

We use induction on the order of $G$. Let $N$ be an arbitrary non-trivial normal subgroup of $G$. Clearly, $H N / N$ is normal in $G / N$ and

$$
(G / N) /(H N / N) \cong G /(H N) \cong(G / H) /(H N / H)
$$

is $p$-nilpotent. Besides, by Lemma 2, every maximal subgroup of every Sylow $p$-subgroup of $H N / N$ is normally embedded in $G / N$ and $(|G / N|, p-1)=1$. Hence the quotients $G / N$ satisfy the hypotheses of the theorem.

By the inductive hypothesis, $G / N$ is $p$-supersolvable. By Lemma 3 , $Z(G)=O_{p^{\prime}}(G)=\Phi(G)=1, G$ contains a unique minimal normal subgroup

$$
N=F(G)=O_{p}(G)=C_{G}(N), G=[N] M,
$$


$N$ is an elementary Abelian subgroup of order $p^{n}, n>1, M$ is a maximal subgroup of $G$.

Since $N \leqslant H$, then $N$ is contained in every Sylow $p$-subgroup $P$ of $H$. By Lemma 6, we have a contradiction. The theorem is proved.

Corollary 2. Let $G$ be a group, $H$ be a normal subgroup of group $G$ such that $G / H$ is p-nilpotent and $P$ be a Sylow p-subgroup of $H$, where $p$ is the smallest in $\pi(G)$. If every subgroup of $\mathcal{M}(P)$ is normally embedded in $G$, then $G$ is p-nilpotent.

Corollary 3. Let $G$ be a group and $P$ be a Sylow $p$-subgroup of $G$, where $p \in \pi(G)$ with $(|G|, p-1)=1$. If every subgroup of $\mathcal{M}(P)$ is normally embedded in $G$, then $G$ is p-nilpotent.

Corollary 4. Let $G$ be a group and $P$ be a Sylow $p$-subgroup of $G$, where $p$ is the smallest in $\pi(G)$. If every subgroup of $\mathcal{M}(P)$ is normally embedded in $G$, then $G$ is p-nilpotent.

Corollary 5. Let $G$ be a group. If every subgroup of $\mathcal{M}(G)$ is normally embedded in $G$, then $G$ possesses an ordered Sylow tower of supersolvable type.

Proof. Let $p$ be the smallest prime of $\pi(G)$ and $P$ be a Sylow $p$-subgroup of $G$. Then by hypothesis, every subgroup of $\mathcal{M}(P)$ is normally embedded in $G$. By Corollary $4, G$ is $p$-nilpotent. By Lemma 1 (1) and the inductive hypothesis, a Hall $p^{\prime}$-subgroup of $G$ has an ordered Sylow tower of supersolvable type. Consequently, $G$ has an ordered Sylow tower of supersolvable type.

\section{References}

[1] B. Huppert, Endliche Gruppen I. Berlin-Heidelberg-New York, Springer, 1967.

[2] V.S. Monakhov, Introduction to the Theory of Final Groups and Their Classes [in Russian]. Vysh. Shkola, Minsk, 2006.

[3] S. Srinivasan, Two sufficient conditions for supersolvability of finite groups, Israel J. Math., 35, 1980, pp.210-214.

[4] V.S. Monakhov, A. A. Trofimuk, Finite groups with subnormal non-cyclic subgroups, J. Group Theory, 17(5), 2014, pp.889-895.

[5] W. Guo, Y. Lu, W. Niu, S-embedded subgroups of finite groups, Algebra Logika, 49(4), 2010, pp.433-450.

[6] K. Doerk and T. Hawkes, Finite soluble groups. Berlin-New York: Walter de Gruyter, 1992. 
[7] V.S. Monakhov, I. K. Chirik. On the p-supersolvability of a finite factorizable group with normal factors, Proceedings of the Institute of Mathematics and Mechanics (Trudy Instituta Matematiki I Mekhaniki), 21(3), 2015, pp.256-267.

[8] O. Yu. Schmidt, Groups whose all subgroups are special, Mat.Sb., 31, 1924, pp.366372.

[9] D. Robinson, A course in the theory of groups, 2nd ed., Graduate Texts in Mathematics, Springer-Verlag, New York (1996).

\section{CONTACT INFORMATION}

Alexander Trofimuk Department of Mathematics, Gomel Francisk Skorina State University, Gomel 246019, Belarus

E-Mail(s): alexander.trofimuk@gmail.com

Received by the editors: 21.04.2018. 